\title{
Article \\ Circulating HDL and Non-HDL Associated Apolipoproteins and Breast Cancer Severity
}

\author{
Christine Bobin-Dubigeon ${ }^{1,2}$, Hassan Nazih ${ }^{1}$ (D), Valentin Blanchard ${ }^{3}$ (D), Mikaël Croyal ${ }^{4,5}$ \\ and Jean-Marie Bard 1,2,*(D)
}

1 CRNHO, West Human Nutrition Research Center, Université de Nantes, EA 2160-IUML FR3473 CNRS, F-44035 Nantes, France; christine.bobin-dubigeon@univ-nantes.fr (C.B.-D.); el-hassane.nazih@univ-nantes.fr (H.N.)

2 Institut de Cancérologie de l'Ouest, F-44805 Saint-Herblain, France

3 Department of Medecine, Centre for Heart Lung Innovation, Providence Healthcare Research Institute, St. Paul's Hospital, University of British Columbia, Vancouver, BC V6Z1Y6, Canada; valentin.blanchard@hli.ubc.ca

4 CRNHO, West Human Nutrition Research Center, Université de Nantes, CNRS, INSERM, l'Institut du Thorax, F-44035 Nantes, France; mikael.croyal@univ-nantes.fr

5 CHU Nantes, Inserm, CNRS, SFR Santé, Inserm UMS 016, CNRS UMS 3556, F-44000 Nantes, France

* Correspondence: jean-marie.bard@univ-nantes.fr

Citation: Bobin-Dubigeon, C.; Nazih, H.; Blanchard, V.; Croyal, M.; Bard, J.-M. Circulating HDL and Non-HDL Associated Apolipoproteins and Breast Cancer Severity. J. Clin. Med. 2022, 11, 1345. https://doi.org/ $10.3390 / j \mathrm{~cm} 11051345$

Academic Editor:

Venerando Rapisarda

Received: 19 January 2022

Accepted: 26 February 2022

Published: 28 February 2022

Publisher's Note: MDPI stays neutral with regard to jurisdictional claims in published maps and institutional affiliations.

Copyright: () 2022 by the authors Licensee MDPI, Basel, Switzerland. This article is an open access article distributed under the terms and conditions of the Creative Commons Attribution (CC BY) license (https:// creativecommons.org/licenses/by/ $4.0 /)$.

\begin{abstract}
Plasma lipids are carried within lipoproteins with various apolipoprotein content. This study evaluates the interest of measuring the apolipoproteins of circulating lipoproteins in breast cancer. Patients with early-stage breast cancer $(n=140)$ were included. Tumors differed by the expression of estrogen and progesterone receptor ( $\mathrm{HR}-$ and $\mathrm{HR}+$ for negative and positive expression) and the proliferation marker Ki-67 ( $\leq 20 \%$ or $\geq 30 \%$ ). Apolipoprotein concentrations were determined in plasma, HDL and non-HDL fractions, and results are given in $\mathrm{mg} / \mathrm{dL}$, median (25th-75th). Patients did not differ in their plasma and lipoprotein lipid concentrations. HDL apoC-I and non-HDL apoC-II were reduced (1.34 (1.02-1.80) vs. 1.61 (1.32-2.04), $p=0.04 ; 0.31(0.18-0.65)$ vs. $0.63(0.39-1.02), p=0.01$; respectively), in $\mathrm{RH}-/$ high Ki-67 patients in comparison to $\mathrm{RH}-/$ low Ki-67 patients, while plasma apoD and HDL apoD were higher (3.24 (2.99-4.16) vs. 3.07 (2.39-3.51), $p=0.04 ; 2.74$ (2.36-3.35) vs. 2.45 (2.01-2.99), $p=0.04$; respectively). When $\mathrm{RH}+/$ high Ki-67 patients were compared with $\mathrm{RH}+/$ low Ki-67 patients, HDL apoC-I and HDL apoC-III were higher (1.56 (1.20-1.95) vs. 1.35 $(1.10-1.62), p=0.02 ; 2.80(2.42-3.64)$ vs. 2.38 (1.69-2.96), $p=0.02$; respectively). The distribution of exchangeable apolipoproteins, such as apoC-I, apoC-II, apoC-III, apoD, between lipoproteins is linked to the severity of breast cancer.
\end{abstract}

Keywords: apolipoproteins; lipoproteins; HDL; non-HDL; breast cancer; Ki-67

\section{Introduction}

Among the last years, several reports evidenced that various metabolic disturbances related to obesity may be associated with an increased risk of breast cancer [1,2]. Circulating lipids may be one of the factors that relate to these metabolic abnormalities and the disease risk [3]. Clinical studies looking at the association between circulating cholesterol carried by Low-Density Lipoproteins (LDL) or High-Density Lipoproteins (HDL) with breast cancer have raised conflicting results. Nevertheless, the results of large studies seem to point towards a positive relationship between LDL and breast cancer, while HDL would rather be negatively associated with the disease [4]. Apolipoproteins play essential roles in maintaining the structural integrity and functional specificity of plasma lipoproteins. They are directly involved in various metabolic processes of lipoproteins, including secretion, prevention of premature removal from the circulation, binding with cell-surface receptors and activation of lipolytic enzymes [5]. Besides their role in lipoprotein metabolism, 
apolipoproteins were shown to be involved in the development of breast cancer, as nicely reviewed recently [6]. The plasma level of some apolipoproteins was related to breast cancer severity. For instance, low levels of plasma apoA-I were shown to independently predict the poor clinical outcome of patients with invasive ductal breast cancer [7]. In another study, lower apoC-I and apoC-II concentrations were found in breast cancer patients when compared to controls, while apoC-III concentration was higher [8]. Women with advanced breast cancer were shown to have higher plasma concentrations of apoD [9]. The apoE plasma concentration was also positively associated with breast cancer malignancy [10]. It is known that apo $\mathrm{E}$ is present in plasma in a polymorphic form. The major isoforms are apoE2, apoE3 and apoE4, leading to six phenotypes, depending on the inherited $\varepsilon$ alleles. There are some studies in the literature suggesting a link between the presence of the $\varepsilon 4$ allele and breast cancer [11]. Therefore, it would be of great interest to consider not only the cholesterol content of lipoproteins but also their apolipoprotein composition when studying the association of circulating lipoproteins with breast cancer. The present study was undertaken to evaluate the interest of measuring the apolipoprotein content of circulating lipoproteins in the context of breast cancer.

\section{Materials and Methods}

\subsection{Patient Samples}

This study included 140 patients with early-stage breast cancer referred to our hospital (ICO René Gauducheau, Saint-Herblain, France). Biological material was collected from our biobank, which was declared to and authorized by the French Research Ministry (Declaration Number: DC-2018-3321). This declaration includes approval by a research ethics committee (CPP: Comité de Protection des Personnes) [12]. Informed consent was obtained from patients, granting permission to use their biological specimens and clinicalpathological data for research purposes, as required by the French legislation and the French committee for the protection of human rights. Patient tumors differed by the expression of estrogen (ER) and / or progesterone (PR) receptor (HR- for ER- PR- and HR+ for ER+ and/or PR+, respectively) and the level of the proliferation marker Ki-67 (Ki-67 $\leq 20 \%$ or $\mathrm{Ki}-67 \geq 30 \%$ ). Although the cut-off value of $20 \%$ is commonly used in clinical practice to categorize patients with high or low proliferative index, the value of $30 \%$ was chosen in order to avoid the "grey zone", which could be misleading for our analyses. Serum and EDTA plasma samples from patients were collected at the time of diagnosis and before any therapeutic intervention, were retrieved from our biobank. The distribution of these sera was as follows: 92 were obtained from $\mathrm{HR}+$ patients, and 48 were obtained from $\mathrm{HR}$ - patients. Among the $\mathrm{HR}+$ group, 41 tumors were $\mathrm{Ki}-67 \geq 30 \%$, and 51 tumors were $\mathrm{Ki}-67 \leq 20 \%$. Among the HR - group, 29 tumors were Ki-67 $\geq 30 \%$, and 19 tumors were $\mathrm{Ki}-67 \leq 20 \%$.

\subsection{Biological Analyses}

Plasma cholesterol, plasma triglycerides, HDL cholesterol and LDL cholesterol concentrations were measured using enzymatic kits from Diasys, according to the manufacturer's instructions (Grabels, France). HDL and non-HDL fractions were separated from EDTA plasma by the specific precipitation of apoB-containing lipoproteins. An $\mathrm{MgCl}_{2}$ solution $(2 \mathrm{~mol} / \mathrm{L}, 2.5 \mu \mathrm{L})$ was added to plasma samples $(100 \mu \mathrm{L})$. Then, $10 \mu \mathrm{L}$ of $4 \%$ phosphotungstic acid (in $\mathrm{NaOH} 1 \mathrm{~mol} / \mathrm{L}$ :water; 16:84; v:v) were added. Samples were vortex-mixed and centrifuged for $30 \mathrm{~min}\left(4^{\circ} \mathrm{C}, 4000 \times g\right)$. Supernatants (HDL fraction) were collected, and the pellets (non-HDL fraction) were resuspended in $100 \mu \mathrm{L}$ of ultra-pure water. Total plasma, HDL and non-HDL apolipoproteins A-I, B100, C-I, C-II, C-III, D and E, as well as apoE phenotyping, were determined by liquid chromatography-tandem mass spectrometry, as described previously [13]. Briefly, the apolipoproteins were quantified in $40 \mu \mathrm{L}$ sample aliquots using trypsin proteolysis and the subsequent analysis of proteotypic peptides. The intra- and inter-assay variabilities did not exceed $6.5 \%$ in plasma and HDL samples. The non-HDL concentrations were deducted by subtracting total plasma and 
HDL concentrations. The calculated non-HDL concentrations were compared to the direct measurement of apolipoproteins in the precipitated non-HDL fraction in a representative set of samples $(25 \%)$. Since coefficients of variation did not exceed $6.8 \%$ between both measurements, we kept the calculated non-HDL values for analyses to gain sensitivity.

\subsection{Statistical Analyses}

All statistics were calculated using SAS software (Chapel Hill, NC, USA) version 9.04. The univariate procedure was also used to determine median and 25th and 75th intervals for these variables. The statistical differences between biological variables in EDTA plasma, HDL and non-HDL between patients with different levels of proliferation were estimated by median test analyses in each subset of samples, HR+ and HR-.

\section{Results}

Table 1 presents the clinical characteristics of the studied population. HR+ patients were significantly older $(p=0.003)$ in the group with low proliferative index, explaining the difference in menopausal status $(p=0.006)$. However, no difference in BMI and normolipidemic drug consumption were observed. Infiltrating duct carcinoma was the most common type of cancer found. As expected, the histopronostic grade of tumors differed according to the $\mathrm{HR} / \mathrm{Ki}-67$ groups with the most pejorative grade in the $\mathrm{Ki}-67 \geq 30$ both for HR+ and HR - groups $(p<0.001)$. Tumors of all HR+ patients with a high Ki-67 level were luminal B HER2- 26/41 (63.4\%) or HER2+ 15/41 (36.6\%), while in the sub-group of low Ki-67 level, 46/51 (90.2\%) were luminal A and 5/51 (9.8\%) were luminal B HER2+. For HR- patients, $21 / 29$ patients $(72.4 \%)$ were triple-negative breast cancer (TNBC) in the sub-group of high Ki-67 level, and 16/19 (84.2\%) were TNBC in the sub-group of low Ki-67 level.

Table 1. Clinicobiological parameters of the studied cohort. Data are presented with median (25th-75th percentile) and frequencies as number (precentage).

\begin{tabular}{|c|c|c|c|c|c|c|}
\hline & \multicolumn{3}{|c|}{$\mathbf{H R}-$} & \multicolumn{3}{|c|}{ HR+ } \\
\hline & $\begin{array}{c}\mathrm{Ki}-67 \leq 20 \% \\
\quad(n=19)\end{array}$ & $\begin{array}{c}\mathrm{Ki}-67 \geq 30 \% \\
\quad(n=29)\end{array}$ & $p$ & $\begin{array}{c}\mathrm{Ki}-67 \leq 20 \% \\
\quad(n=51)\end{array}$ & $\begin{array}{c}\mathrm{Ki}-67 \geq 30 \% \\
\quad(n=41)\end{array}$ & $p$ \\
\hline $\begin{array}{l}\text { Age } \\
\text { (years) }\end{array}$ & $\begin{array}{c}62.5 \\
{[56.0-69.0]}\end{array}$ & $\begin{array}{c}54.4 \\
{[43.0-66.0]}\end{array}$ & 0.08 & $\begin{array}{c}64.1 \\
{[56.0-72.5]}\end{array}$ & $\begin{array}{c}56.2 \\
{[48-66.0]}\end{array}$ & 0.003 \\
\hline $\begin{array}{l}\mathrm{BMI} \\
\left(\mathrm{Kg} / \mathrm{m}^{2}\right)\end{array}$ & $\begin{array}{c}24.7 \\
{[21.8-27.3]}\end{array}$ & $\begin{array}{c}25.2 \\
{[22.2-27.1]}\end{array}$ & 0.66 & $\begin{array}{c}25.4 \\
{[22.7-27.5]}\end{array}$ & $\begin{array}{c}26.1 \\
{[21.9-28.8]}\end{array}$ & 0.67 \\
\hline Menopause & & & 0.15 & & & 0.006 \\
\hline Yes & $17(89 \%)$ & $21(72 \%)$ & & $43(84.3 \%)$ & $24(58.8 \%)$ & \\
\hline No & $2(11 \%)$ & $8(28 \%)$ & & $8(15.7 \%)$ & $17(41.2 \%)$ & \\
\hline $\begin{array}{l}\text { Normolipidemic } \\
\text { Treatment }\end{array}$ & & & 0.85 & & & 0.71 \\
\hline Yes & $3(16 \%)$ & $4(14 \%)$ & & $5(9.8 \%)$ & $5(12 \%)$ & \\
\hline No & $16(84 \%)$ & $25(86 \%)$ & & $46(90.2 \%)$ & $36(88 \%)$ & \\
\hline Type of cancer & & & 0.29 & & & 0.01 \\
\hline $\begin{array}{l}\text { Inflitrating duct } \\
\text { carcinoma }\end{array}$ & $16(84 \%)$ & $28(97 \%)$ & & $51(100 \%)$ & $36(88 \%)$ & \\
\hline $\begin{array}{l}\text { Invasive lobular } \\
\text { carcinoma }\end{array}$ & $2(11 \%)$ & $1(3 \%)$ & & $0(0 \%)$ & $5(12 \%)$ & \\
\hline Histopronostic grade & & & $<0.001$ & & & $<0.001$ \\
\hline Grade I & $0(0 \%)$ & $0(0 \%)$ & & $21(41.2 \%)$ & $0(0 \%)$ & \\
\hline Grade II & $17(89 \%)$ & $3(10.3 \%)$ & & $26(51 \%)$ & $6(14.6 \%)$ & \\
\hline Grade III & $2(11 \%)$ & $26(89.7 \%)$ & & $4(7.8 \%)$ & $35(85.4 \%)$ & \\
\hline Molecular sub-types & & & & & & \\
\hline Luminal B HER2+ & & & & $5(9.8 \%)$ & $15(36.6 \%)$ & \\
\hline Luminal B HER2- & & & & $0(0 \%)$ & $26(63.4 \%)$ & $<0.001$ \\
\hline Luminal A & & & & $46(90.2 \%)$ & $0(0 \%)$ & \\
\hline TNBC & $16(84.2 \%)$ & $21(72.4 \%)$ & & & & \\
\hline HER2 Type & $3(15.8 \%)$ & $8(27.6 \%)$ & 0.488 & & & \\
\hline
\end{tabular}


Table 2 shows the concentration of plasma and lipoprotein lipids in RH- and RH+ breast cancer patients and low (Ki-67 $\leq 20 \%$ ) or high (Ki-67 $\geq 30 \%$ ) proliferative index. In each group of hormone receptor breast cancer patients, no significant difference was observed in any plasma or lipoprotein lipid concentrations between patients with a low proliferative index and patients with a high proliferative index.

Table 2. Plasma and lipoprotein lipids in estrogen receptor-positive $(\mathrm{HR}+)$ and negative $(\mathrm{HR}-)$ breast cancer patients, according to the proliferation index (Ki-67 $\leq 20 \%$ or Ki-67 $\geq 30 \%$ ). Data are presented with median (25th-75th percentile).

\begin{tabular}{|c|c|c|c|c|c|c|}
\hline \multirow[b]{2}{*}{ Parameters } & \multicolumn{2}{|c|}{ HR - } & \multicolumn{4}{|c|}{ HR+ } \\
\hline & $\begin{array}{c}\text { Ki-67 } \leq 20 \% \\
(n=19)\end{array}$ & $\begin{array}{c}\mathrm{Ki}-67 \geq 30 \% \\
\quad(n=29)\end{array}$ & $p$ & $\begin{array}{c}\mathrm{Ki}-67 \leq 20 \% \\
\quad(n=51)\end{array}$ & $\begin{array}{c}\mathrm{Ki}-67 \geq 30 \% \\
\quad(n=41)\end{array}$ & $p$ \\
\hline $\begin{array}{l}\text { Plasma Cholesterol } \\
(\mathrm{mmol} / \mathrm{L})\end{array}$ & $\begin{array}{c}5.30 \\
{[4.53-5.93]}\end{array}$ & $\begin{array}{c}5.25 \\
{[4.07-5.75]}\end{array}$ & 0.770 & $\begin{array}{c}5.03 \\
{[4.17-5.83]}\end{array}$ & $\begin{array}{c}5.36 \\
{[4.44-5.91]}\end{array}$ & 0.297 \\
\hline $\begin{array}{l}\text { Plasma Triglycerides } \\
(\mathrm{mmol} / \mathrm{L})\end{array}$ & $\begin{array}{c}1.23 \\
{[0.91-1.74]}\end{array}$ & $\begin{array}{c}1.11 \\
{[0.79-1.37]}\end{array}$ & 0.381 & $\begin{array}{c}0.90 \\
{[0.71-1.25]}\end{array}$ & $\begin{array}{c}0.92 \\
{[0.74-1.12]}\end{array}$ & 0.835 \\
\hline $\begin{array}{l}\text { LDL Cholesterol } \\
(\mathrm{mmol} / \mathrm{L}\end{array}$ & $\begin{array}{c}3.30 \\
{[2.47-3.82]}\end{array}$ & $\begin{array}{c}3.39 \\
{[2.39-4.01]}\end{array}$ & 0.770 & $\begin{array}{c}3.18 \\
{[2.49-3.90]}\end{array}$ & $\begin{array}{c}3.38 \\
{[2.60-3.97]}\end{array}$ & 0.297 \\
\hline $\begin{array}{l}\text { HDL Cholesterol } \\
(\mathrm{mmol} / \mathrm{L})\end{array}$ & $\begin{array}{c}1.48 \\
{[1.10-1.66]}\end{array}$ & $\begin{array}{c}1.39 \\
{[1.07-1.51]}\end{array}$ & 0.144 & $\begin{array}{c}1.30 \\
{[1.09-1.62]}\end{array}$ & $\begin{array}{c}1.38 \\
{[1.14-1.57]}\end{array}$ & 0.297 \\
\hline $\begin{array}{l}\text { Non HDL Cholesterol } \\
(\mathrm{mmol} / \mathrm{L})\end{array}$ & $\begin{array}{c}3.90 \\
{[3.19-4.65]}\end{array}$ & $\begin{array}{c}3.79 \\
{[2.82-4.46]}\end{array}$ & 0.770 & $\begin{array}{c}3.57 \\
{[2.94-4.39]}\end{array}$ & $\begin{array}{c}3.84 \\
{[3.17-4.50]}\end{array}$ & 0.531 \\
\hline
\end{tabular}

Table 3 shows the concentrations of plasma, HDL and non-HDL apolipoproteins. When considering the plasma concentrations, no difference between low proliferative index and the high proliferative index was observed, either in $\mathrm{RH}$ - or $\mathrm{RH}+$ patients, with the exception of apoD, which was significantly increased in $\mathrm{RH}$ - patients with a high $\mathrm{Ki}-67$ index when compared with RH- patients with a low Ki-67 index $(p=0.042)$. The patient populations did not differ for the plasma concentrations of either apoA-I, the main carrier of HDL or apoB100, the main carrier of non-HDL lipoproteins. RH- patients were characterized by a lower concentration of HDL apo-C-I when their tumor exhibited a higher proliferative index $(p=0.041)$, while the opposite was observed for $\mathrm{RH}+$ patients $(p=0.022)$. In $\mathrm{RH}+$ patients, a tendency towards a higher non-HDL apo-C-I concentration $(p=0.060)$ was also observed in the case of a high Ki-67 index. RH- patients with a high $\mathrm{Ki}-67$ index had a lower concentration of non-HDL apoC-II than those with a low Ki-67 index (p0.009). HDL apo-C-III was significantly increased in $\mathrm{RH}+$ patients with a high proliferative index, compared with $\mathrm{RH}+$ patients with a low proliferative index $(p=0.022)$. In RH- patients, HDL apoD was higher in patients with a high Ki-67 index when compared with RH- patients with a low Ki-67 index $(p=0.035)$.

Table 3. Concentration of plasma, HDL and non-HDL apolipoproteins in estrogen receptor-positive $(\mathrm{HR}+)$ and negative (HR-) breast cancer patients, according to the proliferation index (Ki-67 $\leq 20 \%$ or $\mathrm{Ki}-67 \geq 30 \%$ ). Data are presented with median (25th-75th percentile).

\begin{tabular}{|c|c|c|c|c|c|c|}
\hline & \multicolumn{3}{|c|}{ HR - } & \multicolumn{3}{|c|}{$\mathrm{HR}+$} \\
\hline Parameters & $\begin{array}{c}\mathrm{Ki}-67 \leq 20 \% \\
\quad(n=19)\end{array}$ & $\begin{array}{c}\mathrm{Ki}-67 \geq 30 \% \\
\quad(n=29)\end{array}$ & $p$ & $\begin{array}{c}\mathrm{Ki}-67 \leq 20 \% \\
(n=51)\end{array}$ & $\begin{array}{c}\mathrm{Ki}-67 \geq 30 \% \\
\quad(n=41)\end{array}$ & $p$ \\
\hline $\begin{array}{l}\text { Plasma apoA-I } \\
(\mathrm{mg} / \mathrm{dL})\end{array}$ & $\begin{array}{c}148.60 \\
{[133.1-159.0]}\end{array}$ & $\begin{array}{c}160.0 \\
{[137.0-170.0]}\end{array}$ & 0.381 & $\begin{array}{c}152.40 \\
{[128.3-168.4]}\end{array}$ & $\begin{array}{c}148.84 \\
{[133.7-163.6]}\end{array}$ & 0.531 \\
\hline $\begin{array}{l}\text { Plasma apoB100 } \\
(\mathrm{mg} / \mathrm{dL})\end{array}$ & $\begin{array}{c}79.86 \\
{[68.3-104.4]}\end{array}$ & $\begin{array}{c}96.60 \\
{[78.1-117.0]}\end{array}$ & 0.144 & $\begin{array}{c}95.67 \\
{[76.5-118.8]}\end{array}$ & $\begin{array}{c}88.40 \\
{[75.9-108.8]}\end{array}$ & 0.297 \\
\hline $\begin{array}{l}\text { Plasma apoC-I } \\
(\mathrm{mg} / \mathrm{dL})\end{array}$ & $\begin{array}{c}2.15 \\
{[2.00-2.89]}\end{array}$ & $\begin{array}{c}2.46 \\
{[1.78-2.82]}\end{array}$ & 0.381 & $\begin{array}{c}1.99 \\
{[1.64-2.58]}\end{array}$ & $\begin{array}{c}2.30 \\
{[1.97-3.06]}\end{array}$ & 0.060 \\
\hline $\begin{array}{l}\text { HDL apoC-I } \\
\text { (mg/dL) }\end{array}$ & $\begin{array}{c}1.61 \\
{[1.32-2.04]}\end{array}$ & $\begin{array}{c}1.34 \\
{[1.02-1.80]}\end{array}$ & 0.041 & $\begin{array}{c}1.35 \\
{[1.10-1.62]}\end{array}$ & $\begin{array}{c}1.56 \\
{[1.20-1.95]}\end{array}$ & 0.022 \\
\hline
\end{tabular}


Table 3. Cont.

\begin{tabular}{|c|c|c|c|c|c|c|}
\hline \multirow[b]{2}{*}{ Parameters } & \multicolumn{3}{|c|}{$\mathbf{H R}-$} & \multicolumn{3}{|c|}{ HR+ } \\
\hline & $\begin{array}{c}\mathrm{Ki}-67 \leq 20 \% \\
\quad(n=19)\end{array}$ & $\begin{array}{c}\mathrm{Ki}-67 \geq 30 \% \\
\quad(n=29)\end{array}$ & $p$ & $\begin{array}{c}\mathrm{Ki}-67 \leq 20 \% \\
\quad(n=51)\end{array}$ & $\begin{array}{c}\mathrm{Ki}-67 \geq 30 \% \\
\quad(n=41)\end{array}$ & $p$ \\
\hline $\begin{array}{l}\text { Non-HDL apoC-I } \\
(\mathrm{mg} / \mathrm{dL})\end{array}$ & $\begin{array}{c}0.62 \\
{[0.11-1.29]}\end{array}$ & $\begin{array}{c}0.87 \\
{[0.43-1.37]}\end{array}$ & 0.381 & $\begin{array}{c}0.58 \\
{[0.26-1.07]}\end{array}$ & $\begin{array}{c}0.77 \\
{[0.43-0.98]}\end{array}$ & 0.060 \\
\hline $\begin{array}{l}\text { Plasma apoC-II } \\
(\mathrm{mg} / \mathrm{dL})\end{array}$ & $\begin{array}{c}1.97 \\
{[1.43-2.45]}\end{array}$ & $\begin{array}{c}2.00 \\
{[1.60-2.30]}\end{array}$ & 0.770 & $\begin{array}{c}2.00 \\
{[1.56-2.55]}\end{array}$ & $\begin{array}{c}2.00 \\
{[1.49-2.70]}\end{array}$ & 0.835 \\
\hline $\begin{array}{l}\text { HDL apoC-II } \\
(\mathrm{mg} / \mathrm{dL})\end{array}$ & $\begin{array}{c}1.31 \\
{[1.00-1.72]}\end{array}$ & $\begin{array}{c}1.48 \\
{[1.19-2.01]}\end{array}$ & 0.381 & $\begin{array}{c}1.67 \\
{[1.16-1.85]}\end{array}$ & $\begin{array}{c}1.73 \\
{[1.10-2.06]}\end{array}$ & 0.531 \\
\hline $\begin{array}{l}\text { Non-HDL apoC-II } \\
(\mathrm{mg} / \mathrm{dL})\end{array}$ & $\begin{array}{c}0.63 \\
{[0.39-1.02]}\end{array}$ & $\begin{array}{c}0.31 \\
{[0.18-0.65]}\end{array}$ & 0.009 & $\begin{array}{c}0.47[ \\
0.17-0.74]\end{array}$ & $\begin{array}{c}0.48 \\
{[0.23-0.71]}\end{array}$ & 0.835 \\
\hline $\begin{array}{l}\text { Plasma apoC-III } \\
(\mathrm{mg} / \mathrm{dL})\end{array}$ & $\begin{array}{c}4.54 \\
{[3.77-5.31]}\end{array}$ & $\begin{array}{c}4.20 \\
{[3.70-5.10]}\end{array}$ & 0.144 & $\begin{array}{c}4.49 \\
{[3.80-5.33]}\end{array}$ & $\begin{array}{c}4.94 \\
{[3.92-6.04]}\end{array}$ & 0.297 \\
\hline $\begin{array}{l}\text { HDL apoC-III } \\
\text { (mg/dL) }\end{array}$ & $\begin{array}{c}2.66 \\
{[2.22-3.12]}\end{array}$ & $\begin{array}{c}2.60 \\
{[2.00-3.30]}\end{array}$ & 0.381 & $\begin{array}{c}2.38 \\
{[1.69-2.96]}\end{array}$ & $\begin{array}{c}2.80 \\
{[2.42-3.64]}\end{array}$ & 0.022 \\
\hline $\begin{array}{l}\text { Non-HDL apoC-III } \\
(\mathrm{mg} / \mathrm{dL})\end{array}$ & $\begin{array}{c}1.98 \\
{[1.31-3.13]}\end{array}$ & $\begin{array}{c}1.87 \\
{[1.07-2.71]}\end{array}$ & 0.381 & $\begin{array}{c}2.14 \\
{[1.29-2.95]}\end{array}$ & $\begin{array}{c}1.99 \\
{[1.40-2.74]}\end{array}$ & 0.835 \\
\hline $\begin{array}{l}\text { Plasma apoD } \\
(\mathrm{mg} / \mathrm{dL})\end{array}$ & $\begin{array}{c}3.07 \\
{[2.39-3.51]}\end{array}$ & $\begin{array}{c}3.24 \\
{[2.99-4.16]}\end{array}$ & 0.042 & $\begin{array}{c}3.61 \\
{[3.09-4.27]}\end{array}$ & $\begin{array}{c}3.40 \\
{[2.69-4.06]}\end{array}$ & 0.233 \\
\hline $\begin{array}{l}\text { HDL apoD } \\
(\mathrm{mg} / \mathrm{dL})\end{array}$ & $\begin{array}{c}2.45 \\
{[2.01-2.99]}\end{array}$ & $\begin{array}{c}2.74 \\
{[2.36-3.35]}\end{array}$ & 0.035 & $\begin{array}{c}2.88 \\
{[2.48-3.43]}\end{array}$ & $\begin{array}{c}2.63 \\
{[2.22-3.21]}\end{array}$ & 0.156 \\
\hline $\begin{array}{l}\text { Non-HDL apoD } \\
(\mathrm{mg} / \mathrm{dL})\end{array}$ & $\begin{array}{c}0.48 \\
{[0.38-0.65]}\end{array}$ & $\begin{array}{c}0.57 \\
{[0.37-0.69]}\end{array}$ & 0.300 & $\begin{array}{c}0.64 \\
{[0.51-0.91]}\end{array}$ & $\begin{array}{c}0.73 \\
{[0.49-0.96]}\end{array}$ & 0.903 \\
\hline $\begin{array}{l}\text { Plasma apoE } \\
(\mathrm{mg} / \mathrm{dL})\end{array}$ & $\begin{array}{c}6.07[ \\
5.20-7.54]\end{array}$ & $\begin{array}{c}5.71 \\
{[4.64-7.48]}\end{array}$ & 0.770 & $\begin{array}{c}6.11 \\
{[5.06-7.68]}\end{array}$ & $\begin{array}{c}6.30 \\
{[5.07-7.95]}\end{array}$ & 0.835 \\
\hline $\begin{array}{l}\text { HDL apoE } \\
(\mathrm{mg} / \mathrm{dL})\end{array}$ & $\begin{array}{c}2.75 \\
{[2.18-4.08]}\end{array}$ & $\begin{array}{c}2.99 \\
{[1.96-3.50]}\end{array}$ & 0.144 & $\begin{array}{c}2.80 \\
{[2.25-4.82]}\end{array}$ & $\begin{array}{c}3.32 \\
{[1.78-4.64]}\end{array}$ & 0.297 \\
\hline $\begin{array}{l}\text { Non-HDL apoE } \\
(\mathrm{mg} / \mathrm{dL})\end{array}$ & $\begin{array}{c}3.68 \\
{[2.31-4.42]}\end{array}$ & $\begin{array}{c}3.10 \\
{[2.40-4.70]}\end{array}$ & 0.381 & $\begin{array}{c}3.11 \\
{[1.90-4.23]}\end{array}$ & $\begin{array}{c}3.17 \\
{[2.63-3.96]}\end{array}$ & 0.531 \\
\hline
\end{tabular}

In order to determine if the observed differences were related to a difference in the absolute concentration of HDL or non-HDL lipoproteins carrying a given apolipoprotein or if they were related to a relative enrichment or a relative impoverishment in this apolipoprotein, the molar ratios between these apolipoproteins and apoA-I in HDL or apoB100 in non-HDL were compared between patients with a low proliferative index and patients with a high proliferative index. These results are shown in Table 4. RH- patients were characterized by a decrease in the apoC-I-to-apoA-I ratio in HDL when Ki-67 was high compared with patients with a low Ki-67 index $(p=0.001)$. The opposite was observed in $\mathrm{RH}+$ patients, with higher values of this ratio in patients with a high Ki-67 value $(p=0.007)$ In RH- patients, the apoC-II-to-apoB100 ratio in non-HDL was lower when the Ki-67 was high when compared with the low Ki-67 sub-group $(p=0.001)$. The apoC-III-to-apoA-I ratio of HDL was also lower in RH- patients with a high Ki-67 value when compared with the low Ki-67 sub-group ( $p=0.041)$. In $\mathrm{RH}+$ patients, the apoE-to-apoB100 ratio in non-HDL was significantly higher in the sub-group of patients with a high Ki-67 value, in comparison with patients with a low Ki-67 value $(p=0.022)$. A tendency $(p=0.060)$ towards a higher apo-C-III-to-apoA-I ratio in $\mathrm{HDL}$ of $\mathrm{RH}+$ patients with a high Ki-67 value was also observed. 
Table 4. Molar ratios between apolipoproteins and apoA-I in HDL or apoB100 in non-HDL. Data are presented with median (25th-75th percentile).

\begin{tabular}{|c|c|c|c|c|c|c|}
\hline \multirow[b]{2}{*}{ Molar Ratio } & \multicolumn{3}{|c|}{ HR - } & \multicolumn{3}{|c|}{ HR+ } \\
\hline & $\begin{array}{c}\mathrm{Ki}-67 \leq 20 \% \\
(n=19)\end{array}$ & $\begin{array}{c}\mathrm{Ki}-67 \geq 30 \% \\
\quad(n=29)\end{array}$ & $p$ & $\begin{array}{c}\mathrm{Ki}-67 \leq 20 \% \\
\quad(n=51)\end{array}$ & $\begin{array}{c}\mathrm{Ki}-67 \geq 30 \% \\
\quad(n=41)\end{array}$ & $p$ \\
\hline HDL apoC-I/apoA-I & $\begin{array}{c}0.045 \\
{[0.040-0.054]}\end{array}$ & $\begin{array}{c}0.030 \\
{[0.027-0.042]}\end{array}$ & 0.001 & $\begin{array}{c}0.031 \\
{[0.028-0.039]}\end{array}$ & $\begin{array}{c}0.039 \\
{[0.031-0.050]}\end{array}$ & 0.007 \\
\hline $\begin{array}{l}\text { Non-HDL apoC-I/ } \\
\text { apoB100 }\end{array}$ & $\begin{array}{c}0.56 \\
{[0.12-0.99]}\end{array}$ & $\begin{array}{c}0.64 \\
{[0.30-1.03]}\end{array}$ & 0.381 & $\begin{array}{c}0.46 \\
{[0.24-0.72]}\end{array}$ & $\begin{array}{c}0.64 \\
{[0.32-0.88]}\end{array}$ & 0.144 \\
\hline HDL apoC-II/apoA-I & $\begin{array}{c}0.029 \\
{[0.024-0.037]}\end{array}$ & $\begin{array}{c}0.033 \\
{[0.027-0.036]}\end{array}$ & 0.381 & $\begin{array}{c}0.032 \\
{[0.027-0.038]}\end{array}$ & $\begin{array}{c}0.034 \\
{[0.025-0.042]}\end{array}$ & 0.531 \\
\hline $\begin{array}{l}\text { Non HDL apoC-II/ } \\
\text { apoB100 }\end{array}$ & $\begin{array}{c}0.51 \\
{[0.36-0.78]}\end{array}$ & $\begin{array}{c}0.21 \\
{[0.13-0.48]}\end{array}$ & 0.001 & $\begin{array}{c}0.28 \\
{[0.13-0.40]}\end{array}$ & $\begin{array}{c}0.34 \\
{[0.21-0.45]}\end{array}$ & 0.531 \\
\hline HDL apoC-III/apoA-I & $\begin{array}{c}0.065 \\
{[0.055-0.074]}\end{array}$ & $\begin{array}{c}0.055 \\
{[0.046-0.066]}\end{array}$ & 0.041 & $\begin{array}{c}0.054 \\
{[0.038-0.063]}\end{array}$ & $\begin{array}{c}0.060 \\
{[0.052-0.084]}\end{array}$ & 0.060 \\
\hline $\begin{array}{l}\text { Non HDL apoC-III/ } \\
\text { apoB100 }\end{array}$ & $\begin{array}{c}1.44 \\
{[1.11-2.21]}\end{array}$ & $\begin{array}{c}1.15 \\
{[0.82-1.92]}\end{array}$ & 0.381 & $\begin{array}{c}1.32 \\
{[0.99-1.93]}\end{array}$ & $\begin{array}{c}1.47 \\
{[1.18-1.88]}\end{array}$ & 0.297 \\
\hline HDL apoD/apoA-I & $\begin{array}{c}0.017 \\
{[0.015-0.021]}\end{array}$ & $\begin{array}{c}0.017 \\
{[0.016-0.019]}\end{array}$ & 0.593 & $\begin{array}{c}0.018 \\
{[0.015-0.024]}\end{array}$ & $\begin{array}{c}0.018 \\
{[0.015-0.021]}\end{array}$ & 0.481 \\
\hline $\begin{array}{l}\text { Non HDL apoD/ } \\
\text { apoB100 }\end{array}$ & $\begin{array}{c}0.14 \\
{[0.09-0.15]}\end{array}$ & $\begin{array}{c}0.11 \\
{[0.07-0.16]}\end{array}$ & 0.821 & $\begin{array}{c}0.12 \\
{[0.09-0.19]}\end{array}$ & $\begin{array}{c}0.16 \\
{[0.10-0.21]}\end{array}$ & 0.537 \\
\hline HDL apoE/apoA-I & $\begin{array}{c}0.018 \\
{[0.014-0.023]}\end{array}$ & $\begin{array}{c}0.016 \\
{[0.010-0.019]}\end{array}$ & 0.144 & $\begin{array}{c}0.018 \\
{[0.012-0.023]}\end{array}$ & $\begin{array}{c}0.018 \\
{[0.013-0.023]}\end{array}$ & 0.835 \\
\hline $\begin{array}{l}\text { Non HDL apoE/ } \\
\text { apoB100 }\end{array}$ & $\begin{array}{c}0.66 \\
{[0.43-0.83]}\end{array}$ & $\begin{array}{c}0.51 \\
{[0.41-0.64]}\end{array}$ & 0.144 & $\begin{array}{c}0.48 \\
{[0.36-0.60]}\end{array}$ & $\begin{array}{c}0.58 \\
{[0.38-0.72]}\end{array}$ & 0.022 \\
\hline
\end{tabular}

\section{Discussion}

Here, we aimed to evaluate the interest of measuring the apolipoprotein content within circulating lipoproteins in breast cancer patients. We showed that patients with breast cancer of various severity display similar concentrations of plasma and lipoprotein lipids but different concentrations of some apolipoproteins carried by HDL and non-HDL. Besides, we showed that the relationship between apolipoproteins and the disease severity differs between hormone receptor-negative and hormone receptor-positive tumors. Some previously published results indicated that some apolipoproteins might be involved in the development of breast cancer. These results were nicely summarized in a recent review [6].

Despite the fact that low levels of plasma apoA-I were shown to independently predict the poor clinical outcome of patients with invasive ductal breast cancer [7], a large study conducted on 1411 women from the AMORIS cohort with breast cancer severity known only found a modest positive association between the apoB100-to-apoA-I ratio and breast cancer severity [14]. In line with these previous results, we did not find any difference between patients with high proliferative tumor index and patients with low proliferative tumor index. This was the case for $\mathrm{RH}$ - as well as $\mathrm{RH}+$ tumors.

In a study using serum fractionation by strong anion exchange chromatography followed by mass spectrometry analysis, lower apoC-I and apoC-II concentrations were found in breast cancer patients than controls, while apoC-III concentration was higher [8]. In a recent study comparing protein profiles between breast cancer patients and controls, it was also shown that plasma apoC-I was lower in affected patients, and further analyses identified apoC-I signature peptides able to inhibit breast cancer cell proliferation in vitro [15]. It was also suggested that elevated apoC-I levels could help distinguish triple-negative breast cancer and non-triple negative breast cancer [16]. These results pointed out the potential of apoC-I as a biomarker of breast cancer. However, the design and goals of the present study were different from those published previously. Our results suggest that it may be of interest to distinguish between HDL and non-HDL apolipoproteins when studying the relationship of apolipoproteins with breast cancer. As a matter of fact, in the present study, plasma apoC-I, apoC-II and apoC-III concentrations did not differ between patients with 
high proliferative index tumors and patients with low proliferative index tumors. However, HDL apoC-I was lower in the case of high proliferative index tumors when breast cancer was RH-, while it was higher in high proliferative index tumors when breast cancer was $\mathrm{RH}+$. HDL apoC-III was also higher in $\mathrm{RH}+$ patients with a high proliferative index tumor. This deeply suggests that the relationship between apoC-I or apoC-III and breast cancer tumor behavior concerns only HDL and that it differs from the nature of the tumor. The only difference observed for apoC-II was for non-HDL concentration, which was lower in case of high proliferative index RH- tumors. The analysis of the ratios between one apolipoprotein and apoA-I for HDL or one apolipoprotein and apoB100 for non-HDL may be used to determine if these differences are related to a change in the absolute number of lipoprotein particles containing this apolipoprotein or if this is related to a change in the number of copies of a given apolipoprotein per lipoprotein particle. If this ratio increases, this means that the number of copies of this apolipoprotein per lipoprotein particle increases; if this ratio decreases, this means that the number of copies of this apolipoprotein per lipoprotein particle decreases. This change in the apolipoprotein composition of the lipoprotein particle may be independent of its absolute concentration in plasma but it reflects a modification of its quality, which may affect its biological behavior. Concerning apolipoproteins C-I, C-II and C-III, our results suggest that these differences are due to a change in the number of copies of each apolipoprotein per lipoprotein particle.

ApoD is probably one of the apolipoproteins that were the most extensively studied in the context of breast cancer. However, its interaction with the disease is rather complex. While apoD has been shown to inhibit the proliferation of breast cancer cells [17], estrogens significantly reduce apoD gene expression [18]. At the cell level, apoD may influence several critical pathways, including MAPK, 5-LO and COX-2 pathways [19-22]. It was also suggested that apoD expression could be predictive of breast cancer recurrence in tamoxifen-treated patients [23-28]. However, a large population-based case-control study on 11,251 women with well-characterized tumors failed to demonstrate any association between apoD nuclear and cytoplasmic expression and disease recurrence in HR- as well as tamoxifen-treated HR+ patients [29]. Nevertheless, women with advanced breast cancer were shown to have higher plasma concentrations of apoD [9], and apoD was suggested to be a good prognostic indicator for the disease [30]. In the present study, we showed that plasma apoD is higher in the case of high proliferative index tumors, only in the case of $\mathrm{RH}$ - breast cancer tumors. In addition, this difference was observed for HDL only, while non-HDL apoD did not differ with the proliferative index of tumors. However, when calculating the ratio between apoD and apoA-I in HDL, these differences disappeared, suggesting that the relationship between HDL apoD and RH- breast cancer severity is due to an increased number of HDL particles carrying apoD, and not to a relative enrichment of HDL in apoD.

On the cellular level, apoE may inhibit angiogenesis and the proliferation of breast cancer cells [31,32]. By contrast, in a clinical study, it has been shown that the apoE plasma concentration is positively associated with breast cancer malignancy [10]. In the present results, we were unable to show any difference in plasma, HDL or non-HDL apoE concentration between patients with high proliferative index tumors when compared with patients with low proliferative index tumors. However, when considering the relative enrichment of HDL and non-HDL in apoE, it was found that RH+ patients with high proliferative index tumors exhibit non-HDL lipoprotein particles enriched in apoE, as suggested by the significantly higher apoE-to-apoB100 ratio in this group of patients.

Several isoforms of apoE may be found in Humans. The major isoforms are apoE2, apoE3 and apoE4, leading to six phenotypes, depending on the inherited $\varepsilon$ alleles. There are some studies in the literature suggesting a link between the presence of the $\varepsilon 4$ allele and breast cancer [33-35]. However, a meta-analysis found a significant relationship between the presence of this allele and breast cancer only in the Asian population [11]. The ApoE phenotype influences HDL and non-HDL levels in plasma and the apoE concentration [36]. The apoE distribution between HDL and non-HDL may vary with the $\varepsilon$ alleles, and different 
apoE phenotypes distribute differently between lipoproteins [37]. Therefore, the relative enrichment of non-HDL that we observed in $\mathrm{RH}+$ patients could be related to a different distribution of $\varepsilon$ alleles between patients with high proliferative index tumors and patients with low proliferative index tumors. However, in our population, most of the patients carried the E3/E3 phenotype, and the $\varepsilon$ allele distribution did not differ between groups of patients defined by their hormone receptor status and their proliferative index. Therefore, although we cannot exclude an influence of the apoE polymorphism, it does not explain why non-HDL were relatively enriched in apoE in $\mathrm{RH}+$ patients with high proliferative index tumors.

This descriptive study shows that the distribution of apolipoproteins C-I, C-II, C-III and $\mathrm{D}$ between HDL and non-HDL is linked with the severity of breast cancer, as assessed by $\mathrm{Ki}-67$.

Author Contributions: Conceptualization, C.B.-D., H.N., M.C. and J.-M.B.; methodology, C.B.-D., H.N., M.C. and J.-M.B.; validation, C.B.-D., H.N., M.C. and J.-M.B.; formal analysis, J.-M.B.; investigation, C.B.-D., H.N., V.B. and M.C.; resources C.B.-D., M.C. and J.-M.B.; data curation, C.B.-D., M.C. and J.-M.B.; writing_original draft preparation, J.-M.B.; writing-review and editing, C.B.-D., H.N., M.C. and J.-M.B.; visualization, M.C. and J.-M.B.; supervision, J.-M.B.; project administration, J.-M.B.; funding acquisition, C.B.-D. and J.-M.B. All authors have read and agreed to the published version of the manuscript.

Funding: This research was funded by Ligue contre le Cancer 44, 2017 APOPROLIF and the Groupe Lipides Nutrition (GLN) 2017-PC-7225.

Institutional Review Board Statement: Biological material was collected from the ICO biobank, which was declared to and authorized by the French Research Ministry (Declaration Number: DC2018-3321). This declaration includes approval by a research ethics committee (CPP: Comité de Protection des Personnes).

Informed Consent Statement: Informed consent was obtained from patients, granting permission to use their biological specimens and clinical-pathological data for research purposes, as required by the French legislation and the French committee for the protection of human rights.

Data Availability Statement: Data are available on request.

Conflicts of Interest: The authors declare no conflict of interest.

\section{References}

1. Grundy, S.M. Metabolic Complications of Obesity. ENDO 2000, 13, 155-165. [CrossRef]

2. Yung, R.L.; Ligibel, J.A. Obesity and Breast Cancer: Risk, Outcomes, and Future Considerations. Clin. Adv. Hematol. Oncol. 2016, 14, 790-797.

3. Park, J.; Morley, T.S.; Kim, M.; Clegg, D.J.; Scherer, P.E. Obesity and Cancer-Mechanisms Underlying Tumour Progression and Recurrence. Nat. Rev. Endocrinol. 2014, 10, 455-465. [CrossRef] [PubMed]

4. Cedó, L.; Reddy, S.T.; Mato, E.; Blanco-Vaca, F.; Escolà-Gil, J.C. HDL and LDL: Potential New Players in Breast Cancer Development. J. Clin. Med. 2019, 8, 853. [CrossRef]

5. Alaupovic, P. Significance of Apolipoproteins for Structure, Function, and Classification of Plasma Lipoproteins. Methods Enzym. 1996, 263, 32-60. [CrossRef]

6. Zhou, Y.; Luo, G. Apolipoproteins, as the Carrier Proteins for Lipids, Are Involved in the Development of Breast Cancer. Clin. Transl. Oncol. 2020, 22, 1952-1962. [CrossRef]

7. Lin, X.; Hong, S.; Huang, J.; Chen, Y.; Chen, Y.; Wu, Z. Plasma Apolipoprotein A1 Levels at Diagnosis Are Independent Prognostic Factors in Invasive Ductal Breast Cancer. Discov. Med. 2017, 23, 247-258.

8. Opstal-van Winden, A.W.J.; Beijnen, J.H.; Loof, A.; van Heerde, W.L.; Vermeulen, R.; Peeters, P.H.M.; van Gils, C.H. Search for Breast Cancer Biomarkers in Fractionated Serum Samples by Protein Profiling With SELDI-TOF MS: Protein Profiling in Fractionated Serum Samples. J. Clin. Lab. Anal. 2012, 26, 1-9. [CrossRef]

9. Haagensen, D.E.; Mazoujian, G.; Holder, W.D.; Kister, S.J.; Wells, S.A. Evaluation of a Breast Cyst Fluid Protein Detectable in the Plasma of Breast Carcinoma Patients. Ann. Surg. 1977, 185, 279-285. [CrossRef] [PubMed]

10. Xu, X.; Wan, J.; Yuan, L.; Ba, J.; Feng, P.; Long, W.; Huang, H.; Liu, P.; Cai, Y.; Liu, M.; et al. Serum Levels of Apolipoprotein E Correlates with Disease Progression and Poor Prognosis in Breast Cancer. Tumour. Biol. 2016, 37, 15959-15966. [CrossRef]

11. Saadat, M. Apolipoprotein E (APOE) Polymorphisms and Susceptibility to Breast Cancer: A Meta-Analysis. Cancer Res. Treat. 2012, 44, 121-126. [CrossRef] [PubMed] 
12. Heymann, D.; Kerdraon, O.; Verriele, V.; Verhille, E.; Veron, V.; Vitre, M.; Delmas, F.; Henry, C.; Gouy, Y.; Amiand, M.; et al. Centre de Ressources Biologiques-Tumorothèque: Bioresources and Associated Clinical Data Dedicated to Translational Research in Oncology at the Institut de Cancérologie de l'Ouest, France. Open J. Bioresour. 2020, 7, 5. [CrossRef]

13. Blanchard, V.; Garçon, D.; Jaunet, C.; Chemello, K.; Billon-Crossouard, S.; Aguesse, A.; Garfa, A.; Famchon, G.; Torres, A.; Le May, C.; et al. A High-Throughput Mass Spectrometry-Based Assay for Large-Scale Profiling of Circulating Human Apolipoproteins. J. Lipid Res. 2020, 61, 1128-1139. [CrossRef] [PubMed]

14. Melvin, J.C.; Garmo, H.; Holmberg, L.; Hammar, N.; Walldius, G.; Jungner, I.; Lambe, M.; Van Hemelrijck, M. Glucose and Lipoprotein Biomarkers and Breast Cancer Severity Using Data from the Swedish AMORIS Cohort. BMC Cancer 2017, 17, 246. [CrossRef]

15. Sun, Y.; Zhang, J.; Guo, F.; Zhao, W.; Zhan, Y.; Liu, C.; Fan, Y.; Wang, J. Identification of Apolipoprotein C-I Peptides as a Potential Biomarker and Its Biological Roles in Breast Cancer. Med. Sci. Monit. 2016, 22, 1152-1160. [CrossRef]

16. Song, D.; Yue, L.; Zhang, J.; Ma, S.; Zhao, W.; Guo, F.; Fan, Y.; Yang, H.; Liu, Q.; Zhang, D.; et al. Diagnostic and Prognostic Significance of Serum Apolipoprotein C-I in Triple-Negative Breast Cancer Based on Mass Spectrometry. Cancer Biol. 2016, 17, 635-647. [CrossRef]

17. Simard, J.; de Launoit, Y.; Haagensen, D.E.; Labrie, F. Additive Stimulatory Action of Glucocorticoids and Androgens on Basal and Estrogen-Repressed Apolipoprotein-D Messenger Ribonucleic Acid Levels and Secretion in Human Breast Cancer Cells. Endocrinology 1992, 130, 1115-1121. [CrossRef]

18. Simard, J.; Dauvois, S.; Haagensen, D.E.; Lévesque, C.; Mérand, Y.; Labrie, F. Regulation of Progesterone-Binding Breast Cyst Protein GCDFP-24 Secretion by Estrogens and Androgens in Human Breast Cancer Cells: A New Marker of Steroid Action in Breast Cancer. Endocrinology 1990, 126, 3223-3231. [CrossRef]

19. Sivaraman, V.S.; Wang, H.; Nuovo, G.J.; Malbon, C.C. Hyperexpression of Mitogen-Activated Protein Kinase in Human Breast Cancer. J. Clin. Investig. 1997, 99, 1478-1483. [CrossRef]

20. Sarjeant, J.M.; Lawrie, A.; Kinnear, C.; Yablonsky, S.; Leung, W.; Massaeli, H.; Prichett, W.; Veinot, J.P.; Rassart, E.; Rabinovitch, M. Apolipoprotein D Inhibits Platelet-Derived Growth Factor-BB-Induced Vascular Smooth Muscle Cell Proliferated by Preventing Translocation of Phosphorylated Extracellular Signal Regulated Kinase 1/2 to the Nucleus. Arter. Thromb. Vasc. Biol. 2003, 23, 2172-2177. [CrossRef]

21. Romano, M.; Claria, J. Cyclooxygenase-2 and 5-Lipoxygenase Converging Functions on Cell Proliferation and Tumor Angiogenesis: Implications for Cancer Therapy. FASEB J. 2003, 17, 1986-1995. [CrossRef]

22. Matsuyama, M.; Yoshimura, R.; Mitsuhashi, M.; Tsuchida, K.; Takemoto, Y.; Kawahito, Y.; Sano, H.; Nakatani, T. 5-Lipoxygenase Inhibitors Attenuate Growth of Human Renal Cell Carcinoma and Induce Apoptosis through Arachidonic Acid Pathway. Oncol. Rep. 2005, 14, 73-79. [PubMed]

23. Lamelas, M.L.; Vázquez, J.; Enguita, M.I.; Rodríguez, J.C.; González, L.O.; Merino, A.M.; Vizoso, F. Apolipoprotein D Expression in Metastasic Lymph Nodes of Breast Cancer. Int. J. Surg. Investig. 2000, 2, 285-293.

24. Carreño, G.; Del Casar, J.M.; Corte, M.D.; González, L.O.; Bongera, M.; Merino, A.M.; Juan, G.; Obregón, R.; Martínez, E.; Vizoso, F.J. Local Recurrence after Mastectomy for Breast Cancer: Analysis of Clinicopathological, Biological and Prognostic Characteristics. Breast Cancer Res. Treat. 2007, 102, 61-73. [CrossRef]

25. Díez-Itza, I.; Vizoso, F.; Merino, A.M.; Sánchez, L.M.; Tolivia, J.; Fernández, J.; Ruibal, A.; López-Otín, C. Expression and Prognostic Significance of Apolipoprotein D in Breast Cancer. Am. J. Pathol. 1994, 144, 310-320. [PubMed]

26. Do Carmo, S.; Levros, L.-C.; Rassart, E. Modulation of Apolipoprotein D Expression and Translocation under Specific Stress Conditions. Biochim. Biophys. Acta 2007, 1773, 954-969. [CrossRef]

27. Søiland, H.; Skaland, I.; Varhaug, J.E.; Kørner, H.; Janssen, E.A.M.; Gudlaugsson, E.; Baak, J.P.A.; Søreide, J.A. Co-Expression of Estrogen Receptor Alpha and Apolipoprotein D in Node Positive Operable Breast Cancer-Possible Relevance for Survival and Effects of Adjuvant Tamoxifen in Postmenopausal Patients. Acta Oncol. 2009, 48, 514-521. [CrossRef]

28. Søreide, J.A.; Kolnes, J.; Skarstein, A.; Aas, T.; Kvinnsland, S. Progesterone Binding Cyst Protein in Hormone Receptor Positive Breast Cancer; a Predictive Factor for Effect of Adjuvant Tamoxifen Treatment. Anticancer Res. 1994, 14, $2105-2108$.

29. Klebaner, D.; Hamilton-Dutoit, S.; Ahern, T.; Crawford, A.; Jakobsen, T.; Cronin-Fenton, D.P.; Damkier, P.; Janssen, E.; Kjaersgaard, A.; Ording, A.G.; et al. Apolipoprotein D Expression Does Not Predict Breast Cancer Recurrence among Tamoxifen-Treated Patients. PLoS ONE 2017, 12, e0171453. [CrossRef]

30. Lea, O.A.; Kvinnsland, S.; Thorsen, T. Progesterone-Binding Cyst Protein in Human Breast Tumor Cytosol. Cancer Res. 1987, 47, 6189-6192. [PubMed]

31. Campo, G.M.; Avenoso, A.; D'Ascola, A.; Scuruchi, M.; Prestipino, V.; Nastasi, G.; Calatroni, A.; Campo, S. The Inhibition of Hyaluronan Degradation Reduced Pro-Inflammatory Cytokines in Mouse Synovial Fibroblasts Subjected to Collagen-Induced Arthritis. J. Cell. Biochem. 2012, 113, 1852-1867. [CrossRef]

32. El Roz, A.; Bard, J.-M.; Valin, S.; Huvelin, J.-M.; Nazih, H. Macrophage Apolipoprotein E and Proliferation of MCF-7 Breast Cancer Cells: Role of LXR. Anticancer Res. 2013, 33, 3783-3789.

33. Zunarelli, E.; Nicoll, J.A.; Migaldi, M.; Trentini, G.P. Apolipoprotein E Polymorphism and Breast Carcinoma: Correlation with Cell Proliferation Indices and Clinical Outcome. Breast Cancer Res. Treat. 2000, 63, 193-198. [CrossRef]

34. Moysich, K.B.; Freudenheim, J.L.; Baker, J.A.; Ambrosone, C.B.; Bowman, E.D.; Schisterman, E.F.; Vena, J.E.; Shields, P.G Apolipoprotein E Genetic Polymorphism, Serum Lipoproteins, and Breast Cancer Risk. Mol. Carcinog. 2000, 27, 2-9. [CrossRef] 
35. Porrata-Doria, T.; Matta, J.L.; Acevedo, S.F. Apolipoprotein E Allelic Frequency Altered in Women with Early-Onset Breast Cancer. Breast Cancer 2010, 4, 43-48. [CrossRef] [PubMed]

36. Braeckman, L.; De Bacquer, D.; Rosseneu, M.; De Backer, G. Apolipoprotein E Polymorphism in Middle-Aged Belgian Men: Phenotype Distribution and Relation to Serum Lipids and Lipoproteins. Atherosclerosis 1996, 120, 67-73. [CrossRef]

37. Steinmetz, A.; Jakobs, C.; Motzny, S.; Kaffarnik, H. Differential Distribution of Apolipoprotein E Isoforms in Human Plasma Lipoproteins. Arteriosclerosis 1989, 9, 405-411. [CrossRef] [PubMed] 